\title{
Saturation and the integration of Banach valued correspondences ${ }^{\text {it }}$
}

\author{
Yeneng Sun ${ }^{\mathrm{a}, *}$, Nicholas C. Yannelis ${ }^{\mathrm{b}}$ \\ a Departments of Economics and of Mathematics, National University of Singapore, 1 Arts Link, Singapore 117570, Singapore \\ ${ }^{\mathrm{b}}$ Department of Economics, University of Illinois at Urbana-Champaign, Champaign, IL 61820, United States
}

Received 4 December 2006; received in revised form 18 July 2007; accepted 19 July 2007

Available online 19 November 2007

\begin{abstract}
This note illustrates that the saturation property of a probability space can be used to routinely generalize results on the integration of Banach valued correspondences over a Loeb measure space to those over an arbitrary saturated probability space. On the other hand, the saturation property is also necessary for the validity of those results when the target space is infinite dimensional.

(c) 2007 Elsevier B.V. All rights reserved.
\end{abstract}

JEL Classification: C60; C65

Keywords: Saturation; Integration; Correspondences; Banach space; Convexity; Compactness; Upper semicontinuity

\section{Introduction}

In order to study large economies (games) with an infinite dimensional commodity (action) spaces, one needs to work with integration of Banach valued correspondences; see, for example, Rustichini and Yannelis (1991); Sun (1997); Yannelis (1991), and their references. However, it is well known that desirable results such as convexity, compactness and preservation of upper semicontinuity may fail if the underlying measure space is the Lebesgue measure space and the correspondences take values in an infinite dimensional Banach space. To remedy this difficulty, Sun (1997) worked with general correspondences over the Loeb measure spaces as developed in Loeb (1975) while the earlier paper Rustichini and Yannelis (1991) proposed to work with measure spaces whose associated $L^{\infty}$ spaces over any non-null measurable set have strictly larger cardinality than that of the continuum.

The purpose of this note is to illustrate that the saturation property for a probability space as developed in Hoover and Keisler (1984) can be used to routinely generalize results on the integration of Banach valued correspondences over a Loeb measure space to those over an arbitrary saturated probability space. As noted in Keisler and Sun (2002), the saturation property is necessary for those results that are true on a Loeb measure space but fail on a Lebesgue measure space. In our context, it means that in order to obtain the desirable results such as convexity, compactness and preservation of upper semicontinuity for the integration of infinite dimensional Banach valued correspondences, it is necessary to work with saturated probability spaces.

\footnotetext{
We thank a referee for helpful suggestions.

* Corresponding author. Tel.: +65 65163994 .

E-mail addresses: ynsun@nus.edu.sg (Y. Sun), nyanneli@uiuc.edu (N.C. Yannelis).
} 
Corollary 4.5 of Hoover and Keisler (1984) shows that the saturation property of a probability space is equivalent to the $\aleph_{1}$-atomless property. It is also easy to show that a probability space is saturated if and only if its Maharam spectrum is a set of uncountable cardinals; see Fajardo and Keisler (2002) (Theorem 3B.7, p. 47). This latter property of uncountable Maharam spectrum is also called super-atomless in the recent paper Podczeck (2008). The results in Theorems 1, 3, 7 and 8 in Sun (1997) on Loeb spaces are generalized to the setting of a super-atomless measure space ${ }^{1}$, respectively, in Theorems 1-4 in Podczeck (2008). The measurable correspondences in Rustichini and Yannelis (1991) take values within a fixed weakly compact set of a separable Banach space. Podczeck (2008) pushed some ideas used in the proof of the main theorem of Rustichini and Yannelis (1991) in a powerful way so that he could generalize Theorems 1, 2, 7 and 8 in Sun (1996) on convexity, and weak or weak* compactness of (Bochner or Gelfand) integrals of general correspondences over Loeb measure spaces to the setting of a super-atomless measure space. Note that the results in six other parts of Proposition 1 below are not considered in Podczeck (2008).

As noted in Keisler and Sun (2002) and Remark 1 below, one can restate a counterexample on the unit Lebesgue interval to a counterexample on a non-saturated probability space. Thus, the classical Lyapunov example can be used to show that when the target space is the Hilbert space $l_{2}$, each part of Proposition 1 may not hold on a non-saturated probability space. Podczeck (2008) provided stronger counterexamples to show that when the target space is any infinite dimensional Banach space, the convexity and (weak or weak*) compactness of (Bochner or Gelfand) integrals of general correspondences may not hold on a non-saturated probability space.

The rest of the note is organized as follows. Section 2 states the results. We illustrate in Section 3 how the saturation property can be used to routinely generalize results on the integration of Banach valued correspondences over a Loeb measure space to those over an arbitrary saturated probability space.

\section{The results}

Let $X$ and $Y$ be complete separable metric spaces and $\mathcal{M}(X)$ the space of all Borel probability measures on $X$ with the Prohorov metric $\rho$. We recall that $\mathcal{M}(X)$ is again a complete separable metric space. For each $\tau \in \mathcal{M}(X \times Y)$, let $\operatorname{marg}_{X}(\tau)=\tau_{X}$ be the marginal of $\tau$ in $\mathcal{M}(X)$; thus $\operatorname{marg}_{X}: \mathcal{M}(X \times Y) \rightarrow \mathcal{M}(X)$ is a continuous surjection. Let $(\Omega, \mathcal{F}, P)$ be a countably additive complete probability space, $L^{0}(\Omega, X)$ the space of all random variables (measurable functions) $f: \Omega \rightarrow X$ with the metric of convergence in probability.

For $f \in L^{0}(\Omega, X)$, the law (or distribution) of $f$ is defined by law $(f)(B)=P\left(f^{-1}(B)\right.$ ) for each Borel set $B$ in $X$ (law $(f)$ is usually denoted by $P f^{-1}$ in the literature). The law function law : $L^{0}(\Omega, X) \rightarrow \mathcal{M}(X)$ is continuous, and is surjective if $(\Omega, \mathcal{F}, P)$ is atomless.

Definition 1. A probability space $(\Omega, \mathcal{A}, \lambda)$ is saturated or rich if $(\Omega, \mathcal{A}, \lambda)$ is atomless, and for any complete separable metric spaces $X$ and $Y$, any $\tau \in \mathcal{M}(X \times Y)$, any $f \in L^{0}(\Omega, X)$ with law $(f)=\tau_{X}$, there exists $g \in L^{0}(\Omega, Y)$ such that $\operatorname{law}(f, g)=\tau$.

The following proposition generalizes the results in Theorems 1-10 of Sun (1997) on Loeb probability spaces to the case of saturated probability spaces. ${ }^{2}$

Proposition 1. Let $(\Omega, \mathcal{F}, P)$ be a saturated probability space. In parts 1-3 (and parts 4-6) below, X is a (separable) Banach space while the integral is the Bochner integral in the first six parts. In parts 7-10,X is the dual of a separable Banach space and the integral is the Gelfand integral.

1. For any correspondence $F$ from $(\Omega, \mathcal{F}, P)$ to $X$, the Bochner integral $\int_{\Omega} F \mathrm{~d} P$ is convex, where $\int_{\Omega} F \mathrm{~d} P$ is the set of Bochner integrals $\int_{\Omega} f \mathrm{~d} P$ for all Bochner integrable selections $f$ of $F$.

2. Let $F$ be a norm compact valued correspondence from $(\Omega, \mathcal{F}, P)$ to X. If $F$ is integrably bounded by a non-negative real-valued integrable function $\phi$ on $(\Omega, \mathcal{F}, P)$ in the sense that for P-almost all $\omega \in \Omega$, $\sup _{x \in F(\omega)}\|x\| \leq \phi(\omega)$, then $\int_{\Omega} F \mathrm{~d} P$ is norm compact.

\footnotetext{
${ }^{1}$ These generalized results are stated, respectively, in parts 1, 3, 7 and 8 in Proposition 1 below.

${ }^{2}$ We refer to Sun (1997); Yannelis (1991) for various other definitions.
} 
3. For any integrably bounded, weakly compact valued correspondence $F$ from $(\Omega, \mathcal{F}, P)$ to $X, \int_{\Omega} F \mathrm{~d} P$ is weakly compact.

4. For any measurable, integrably bounded, weakly compact valued correspondence $F$ from $(\Omega, \mathcal{F}, P)$ to $X$,

$$
\int_{\Omega} \overline{\operatorname{co}} F \mathrm{~d} P=\int_{\Omega} F \mathrm{~d} P
$$

where $\overline{\mathrm{co}} F$ is the correspondence such that for each $\omega \in \Omega, \overline{\mathrm{co}} F(\omega)$ is the norm closure of the convex hull of $F(\omega)$.

5. Let $Y$ be a metric space and $F$ a norm compact valued correspondence from $\Omega \times Y$ to X. Assume that for each fixed $y \in Y, F(\cdot, y)$ is a measurable correspondence from $\Omega$ to $X$, and there is an integrably bounded, norm compact valued correspondence $G$ such that for every $y \in Y, F(\omega, y) \subseteq G(\omega)$ for P-almost all $\omega \in \Omega$. If $F(\omega, y)$ is norm upper semicontinuous on $Y$ for each fixed $\omega$, then $\int_{\Omega} F(\omega, y) \mathrm{d} P(\omega)$ is norm upper semicontinuous on $Y$.

6. Let $Y$ be a metric space and $F$ a weakly compact valued correspondence from $\Omega \times Y$ to $X$. Assume that for each fixed $y \in Y, F(\cdot, y)$ is a measurable correspondence from $\Omega$ to $X$, and there is an integrably bounded, weakly compact valued correspondence $G$ such that for every $y \in Y, F(\omega, y) \subseteq G(\omega)$ for P-almost all $\omega \in \Omega$. If $F(\omega, y)$ is weakly upper semicontinuous on $Y$ for each fixed $\omega$, then $\int_{\Omega} F(\omega, y) \mathrm{d} P(\omega)$ is weakly upper semicontinuous on $Y$.

7. For any correspondence $F$ from $(\Omega, \mathcal{F}, P)$ to $X$, the Gelfand integral $\int_{\Omega} F \mathrm{~d} P$ is convex, where $\int_{\Omega} F \mathrm{~d} P$ is the set of the Gelfand integrals $\int_{\Omega} f \mathrm{~d} P$ for all Gelfand integrable selections $f$ of $F$.

8. For any integrably bounded, weak ${ }^{*}$ compact valued correspondence $F$ from $(\Omega, \mathcal{F}, P)$ to $X$, the Gelfand integral $\int_{\Omega} F \mathrm{~d} P$ is weak ${ }^{*}$ compact.

9. For any measurable, integrably bounded, weak ${ }^{*}$ compact valued correspondence $F$ from $(\Omega, \mathcal{F}, P)$ to $X$,

$$
\int_{\Omega} w^{*}-\overline{\mathrm{co}} F \mathrm{~d} P=\int_{\Omega} F \mathrm{~d} P
$$

where $w^{*}-\overline{\mathrm{co}} F$ is the correspondence such that for each $\omega \in \Omega, w^{*}-\overline{\mathrm{co}} F(\omega)$ is the weak ${ }^{*}$ closure of the convex hull of $F(\omega)$.

10. Let $Y$ be a metric space and $F$ a weak* compact valued correspondence from $\Omega \times Y$ to $X$. Assume that for each fixed $y \in Y, F(\cdot, y)$ is a weak ${ }^{*}$ measurable correspondence from $\Omega$ to $X$, and there is a real valued integrable function $g$ on $(\Omega, \mathcal{F}, P)$ such that for $P$-almost all $\omega \in \Omega$, $\sup \{\|x\|: x \in F(\omega, y)$ forsomey $\in Y\} \leq g(\omega)$. If $F(\omega, y)$ is weak ${ }^{*}$ upper semicontinuous on $Y$ for each fixed $\omega$, then $\int_{\Omega} F(\omega, y) \mathrm{d} P(\omega)$ is weak ${ }^{*}$ upper semicontinuous on $Y^{3}$

\section{Remark 1.}

(1) The classical example of Lyapunov or its variations can be used to show that all the results in Proposition 1 may fail if $X$ is infinite dimensional and the underlying probability space is the unit Lebesgue interval ${ }^{4}$; see, for example, Sun (1997); Yannelis (1991).

(2) As noted in Fajardo and Keisler (2002); Hoover and Keisler (1984); Keisler and Sun (2002), if $(\Omega, \mathcal{F}, P$ ) is an atomless non-saturated probability space, then there is a set $A \in \mathcal{F}$ such that $P(A)>0$ such that $\mathcal{F}^{A}=\{B \in \mathcal{F}: B \subseteq$ $A\}$ is countably generated. Let $P^{A}$ be the probability measure on $\left(A, \mathcal{F}^{A}\right)$ rescaled from $P$. There is a measurable mapping $h$ from $A$ to $[0,1]$ such that $h$ induces an isomorphism between the corresponding measure algebras of $\left(A, \mathcal{F}^{A}, P^{A}\right)$ and the Lebesgue interval $([0,1], \mathcal{B}, \mu){ }^{5}$ Thus, one can restate a counterexample on the unit Lebesgue interval to a counterexample on a non-saturated probability space through $h$. To illustrate the point, we consider the Lyapunov example and follow the notation in Example 1 of Sun (1997). Let $f$ be the Bochner integrable function

\footnotetext{
${ }^{3}$ When $Y$ is taken to be the set $\{0,1,1 / 2, \ldots, 1 / n, \ldots\}$, the type of results on upper semicontinuity in parts $5,6,10$ are also called Fatou's Lemma in the literature for a sequence of correspondences or functions; see, for example, Sun (1997); Yannelis (1991) and their references.

${ }^{4}$ Similar counterexamples in an arbitrarily given infinite dimensional Banach space are constructed in Podczeck (2008).

5 As noted in Keisler and Sun (2002), this follows from Theorem 4.12, p. 937 of Fremlin (1989).
} 
from the unit Lebesgue interval $([0,1], \mathcal{L}([0,1]), \lambda)$ to the Hilbert space $l_{2}$. Then, there does not exist a Lebesgue measurable subset $E$ of $[0,1]$ such that $\int_{E} f \mathrm{~d} \lambda=1 / 2 \int_{[0,1]} f \mathrm{~d} \lambda$. Let $g$ be an $\mathcal{F}$-measurable mapping from $\Omega$ to $l_{2}$ by letting $g(\omega)=f(h(\omega))$ for $\omega \in A$ and $g(\omega)=0$ for $\omega \notin A$. Suppose that there is an $\mathcal{F}$-measurable set $B$ such that $\int_{B} g \mathrm{~d} P=1 / 2 \int_{\Omega} g \mathrm{~d} P$. Without loss of generality, assume $B \subseteq A$, and let $C$ be the measurable set $h(B)$. Then, $\int_{B} f(h) \mathrm{d} P=1 / 2 \int_{A} f(h) \mathrm{d} P$. Then, $\int_{C} f \mathrm{~d} \lambda=1 / 2 \int_{[0,1]} f \mathrm{~d} \lambda$, which is a contradiction. Hence, the Bochner (Gelfand) integral of the correspondence $G(\omega)=\{0, g(\omega)\}$ is not convex. It is easy to see that the Bochner (Gelfand) integral of the integrably bounded, norm compact valued correspondence $G$ is neither norm closed, nor weakly closed, nor weak* closed. Note that upper semicontinuity also implies closedness. Therefore, each part of Proposition 1 may not hold on the non-saturated probability space $(\Omega, \mathcal{F}, P)$.

(3) Thus, for a general infinite dimensional Banach space that is separable itself or is the dual of a separable Banach space, the saturation property is also necessary for any result of the 10 parts in Proposition 1 to hold on an underlying atomless probability space.

\section{The proofs}

We prove a simple lemma first.

Lemma 1. Let $(\Omega, \mathcal{F}, P)$ and $(T, \mathcal{T}, \lambda)$ be saturated probability spaces. Then, for any norm compact valued, integrably bounded, measurable correspondence $F$ from $(\Omega, \mathcal{F}, P)$ to a separable Banach space $X$, there is a norm compact valued, integrably bounded, measurable correspondence $G$ from $(T, \mathcal{T}, \lambda)$ to $X$ such that $\int_{\Omega} F \mathrm{~d} P=\int_{T} G \mathrm{~d} \lambda$.

Proof. We work with the norm topology first. Let $F$ be any norm compact valued, measurable correspondence from $(\Omega, \mathcal{F}, P)$ to a separable Banach space $X$, which is also integrably bounded by a non-negative real-valued integrable function $\phi$ on $(\Omega, \mathcal{F}, P)$. Let $\mathcal{C}_{X}$ be the space of norm compact subsets of $X$ endowed with the Hausdorff metric, which is also a complete separable metric space. As noted, for example, in Sun (1996), the correspondence $F$ induces a measurable point-valued mapping from $(\Omega, \mathcal{F}, P)$ to $\mathcal{C}_{X}$. Let $\mathcal{H}=\left\{(B, x) \in \mathcal{C}_{X} \times X: x \in B\right\}$, which is closed in the product topology. Then, for a measurable mapping $f$ from $(\Omega, \mathcal{F}, P)$ to $X, f$ is a selection of $F$ if and only if law $(F, f)$ has measure one for the set $\mathcal{H}$.

By the atomlessness property of $(T, \mathcal{T}, \lambda)$, there are measurable mappings $G$ and $\psi$ from $(T, \mathcal{T}, \lambda)$ to $\mathcal{C}_{X}$ and $\mathbb{R}_{+}$, respectively, such that $\operatorname{law}(F, \phi)=\operatorname{law}(G, \psi)$. It follows that $G$ is a norm compact valued, measurable correspondence from $(\Omega, \mathcal{F}, P)$ to $X$ that is integrably bounded by the integrable function $\psi$.

If $f$ is a Bochner integrable selection of $F$, then the saturation property of $(T, \mathcal{T}, \lambda)$ implies that there is a measurable mapping $g$ from $(T, \mathcal{T}, \lambda)$ to $X$ such that $\operatorname{law}(F, f)=\operatorname{law}(G, g)$. Thus, $\operatorname{law}(G, g)(\mathcal{H})=\operatorname{law}(F, f)(\mathcal{H})=1$, which means that $g$ is a measurable selection of $G$. Since $\operatorname{law}(f)=\operatorname{law}(g)$, we know that $g$ is also Bochner integrable with $\int_{\Omega} f \mathrm{~d} P=$ $\int_{T} g \mathrm{~d} \lambda$. This shows that $\int_{\Omega} F \mathrm{~d} P \subseteq \int_{T} G \mathrm{~d} \lambda$. The other side is also clear. Hence $\int_{\Omega} F \mathrm{~d} P=\int_{T} G \mathrm{~d} \lambda$.

Proof of Proposition 1. Let $(T, \mathcal{T}, \lambda)$ be an atomless Loeb probability space, which is saturated by Fajardo and Keisler (2002); Hoover and Keisler (1984); Keisler (1997). Let $F$ and $G$ be the correspondences in the proof of Lemma 1. By Theorems 1 and 2 of Sun (1997), $\int_{T} G \mathrm{~d} \lambda$ is convex and norm compact. Since $\int_{\Omega} F \mathrm{~d} P=\int_{T} G \mathrm{~d} \lambda$ by Lemma 1 , the convexity and norm compactness of $\int_{\Omega} F \mathrm{~d} P$ follows from those of $\int_{T} G \mathrm{~d} \lambda$.

By the proof of Theorems 1 of Sun (1997), the convexity of the Bochner integral of an arbitrary correspondence taking values in a general Banach space follows from the convexity of the Bochner integral of a correspondence consisted of two Bochner integrable functions, which takes values in a separable Banach space and is integrably bounded, norm compact valued and measurable. Hence part 1 follows.

Similarly, the norm compactness of the Bochner integral of a norm compact valued correspondence taking values in a general Banach space follows from the norm compactness of the Bochner integral of an integrably bounded, norm compact valued correspondence that is the closure of a sequence of Bochner integrable functions, which takes values in a separable Banach space and is also measurable. Hence part 2 follows.

Finally, we observe that the proofs of Theorems 1-10 in Sun (1997) only rely on three special results on the distributional properties of correspondences on Loeb spaces developed in Sun (1996), namely, convexity, compactness and a version of preservation of upper semicontinuity, which are stated as Propositions 3.1-3.3 in Sun (1997). However, as noted in Keisler and Sun (2002), all the results on the distributional properties of correspondences on Loeb spaces 
in Sun (1996) can be generalized to results on the distributional properties of correspondences on saturated probability spaces by the saturation property. In particular, Propositions 3.1-3.3 in Sun (1997) are still valid if the underlying probability space is a general saturated probability space. Therefore, exactly the same proof in Sun (1997) can still be used to prove parts 1 and 2 as well as parts $3-10$ of Proposition $1 .^{6}$

\section{References}

Fajardo, S., Keisler, H.J., 2002. Model Theory of Stochastic Processes, Lecture Notes in Logic No. 14. Association for Symbolic Logic, Urbana, IL. Fremlin, D.H., 1989. Measure algebras. In: Handbook of Boolean Algebras, vol. 3. Elsevier, Amsterdam.

Hoover, D.N., Keisler, H.J., 1984. Adapted probability distributions. Transactions of the American Mathematical Society 286, 159-201.

Keisler, H.J., 1997. Rich and saturated adapted spaces. Advances in Mathematics 128, 242-288.

Keisler, H.J., Sun, Y.N., 2002. The necessity of rich probability spaces, mimeo. In: Presented at the 2002 ICM Satellite Confernece Symposium on Stochastics \& Application, Singapore, August 15-17 (http://www.math.nus.edu.sg/ssa/abstracts/YenengSunAbstract.PDF) (Draft paper available at http://www.math.nus.edu.sg/ matsuny/rich.pdf).

Loeb, P.A., 1975. Conversion from nonstandard to standard measure spaces and applications in probability theory. Transactions of the American Mathematical Society 211, 113-122.

Podczeck, K., 2008. On the convexity and compactness of the integral of a Banach space valued correspondence. Journal of Mathematical Economics 44, 836-852.

Rustichini, A., Yannelis, N.C., 1991. What is perfection competition? In: Khan, M.A., Yannelis, N.C. (Eds.), Equilibrium Theory in Infinite Dimensional Spaces. Springer Verlag, Berlin.

Sun, Y.N., 1996. Distributional properties of correspondences on Loeb spaces. Journal of Functional Analysis 139, 68-93.

Sun, Y.N., 1997. Integration of correspondences on Loeb spaces. Transactions of the American Mathematical Society 349, $129-153$.

Yannelis, N.C., 1991. Integration of Banach-valued correspondences. In: Khan, M.A., Yannelis, N.C. (Eds.), Equilibrium Theory in Infinite Dimensional Spaces. Springer Verlag, Berlin.

\footnotetext{
${ }^{6}$ The proofs of Theorems 4 and 9 in Sun (1997) show that parts 4 and 9 of Proposition 1 follow directly from parts 1, 3, 8 of Proposition 1 without further use of the saturation property.
} 